\title{
An Adaptive Algorithm for Image Encryption: Pseudo Random Generator with Crossover of Genetic Algorithm
}

\author{
Hazeem Baqer Taher \\ Computer Science Dept. - Science College - Thi-Qar University
}

\begin{abstract}
In this paper proposed an adaptive approach for image encryption. We used linear congruential random number generators to produce the pseudo random sequence. The input image is encrypted using pseudo random with crossover of genetic algorithm. This new encryption method can be used to encrypt an image with high secure, fast and lossless. It can be used to send the encrypted image through network and secure data storage (such as important documents). To validate our work, several experiments are executed on a number of different features (details) images. The proposed method reduces the weakness area in the encryption method and increasing the randomness of the encrypted image.
\end{abstract}

\footnotetext{
الخلاصة

في هذا البحث نقترح خو ارزميـة مطورة لتشفير الصـور حيث نستخدم crossover of بالاضـافة إلى استخدام pseudo random sequence من اجل انتاج number generator genetic algorithm حيث تم تطوير طريقة لتشفير انواع مختلفـة من الصور لنحصل على صسورة مشفره ذات درجـة تعقيد عاليـة

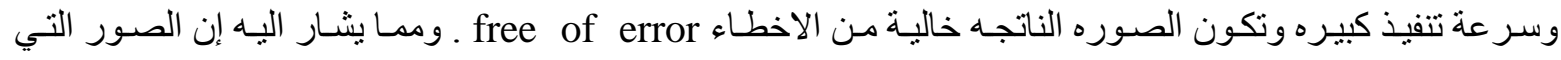
استخدمت كانت تحتوي على كميات مختلفه من التفاصيل. وقد حصلنا على نتائج جيذه حيث قضت هذه الطريقة على نقاط الضعف التي كانت تظهر في حال استخدام طريقة pseudo random وكما ظهر في النتائج.
} 


\section{Introduction}

In the digital world, the security of digital images becomes more and more important since the communications of digital products over open network occur more frequently. Furthermore, special and reliable security in storage and transmission of digital images is needed in many applications, such as pay-TV, medical imaging systems, military image communications, and confidential video conferencing, ect. In order to fulfill such a task, many image encryption methods have been proposed, but some of them have been known to be insecure [1].

Recently, with the greater demand in digital signal transmission and the big losses from illegal data access, data security has become a critical and imperative issue in multimedia data transmission applications. In order to protect valuable information from undesirable readers or against illegal reproduction and modifications, various types of cryptographic schemes are needed. There are two types of cryptographic schemes: symmetric cryptography [2] and asymmetric cryptography [3]. The symmetric scheme uses the same key for encryption and decryption. Two keys issued in asymmetrical cryptography, one for encryption, known as the public key, and the other for decryption, known as the private key. Asymmetric cryptography is often used in key distribution and digital signature for its slow processing speed. The symmetric cryptography is normally used to encrypt private data for its high performance. Moreover, none of the most used symmetrical ciphering systems like DES, IDEA and AES make use of the most recent developments in information processing technology. There have been various data encryption techniques $[4,5]$ on multimedia data proposed in the literature. Genetic Algorithms (GAs) [6] are among such techniques. Generally, genetic algorithms contain three basic operators: reproduction, crossover and mutation where all three are analogous to their namesakes in genetics. Reproduction and crossover together give genetic algorithms most of their searching power.

\section{Pseudo-Random Bit Generators}

It is important to note that the use of pseudo-random sequence generator reduces but does not eliminate the need for a natural source of random bits, the pseudo-random sequence generator is a" randomness expander", but it must be given truly random seed to begin with. A classical techniques for pseudorandom number generation which are quite useful and effective for Monte Carlo simulations are typically unsuitable for cryptographic applications. For example, linear feedback shift registers are well-known to be cryptographically insecure, one can solve for the feedback pattern given a small number of output bits. Linear congruential random number generators are also insecure. These generators use the recurrence:

$\mathrm{X}_{\mathrm{i}+1}=\left(\mathrm{a}_{\mathrm{n}} X_{i}^{n}+\mathrm{a}_{\mathrm{n}-1} X_{i}^{n-1}+\ldots \ldots \ldots+\mathrm{a}_{1} X_{i}+a_{0}\right) \bmod p$

Where $p$ is prime, $a_{n}, \ldots \ldots \ldots, a_{1}, a_{0}$ are integer number less than $\mathrm{p}, \mathrm{x}_{0}$ is initial value for generator. To generate an output sequence $\left\{\mathrm{X}_{1}, \mathrm{X}_{2} \ldots \ldots\right\}$, and starting point $X_{0}$. it is possible to enter the secret parameters given just a few of the $X_{\mathrm{i}}$. even if only a fraction of the bits of each $X_{i}$ are revealed. 


\section{Genetic Algorithms}

A genetic algorithm is a search technique used in finding true or approximate solutions to optimization and search problems. Genetic algorithms are categorized as global search heuristics. Genetic algorithms form a particular class of evolutionary algorithms that use techniques inspired by evolutionary biology such as inheritance, mutation, selection and crossover (also called recombination).Genetic algorithms are implemented as a computer simulation in which a population of abstract representations (called chromosomes or the genotype or the genome) of candidate solutions (called individuals, creatures, or phenotypes) to an optimization problem evolves toward better solutions. Traditionally, solutions are represented in binary strings of 0 s and 1s,but other encodings are also possible. The evolution usually starts from a population of randomly generated individuals. Genetic algorithms form one of the best ways to solve a problem for which a little is known. They are very general algorithms that work well in any search space. A genetic algorithm is able to create a high quality solution [7]. The GA relies primarily on the creative effects of the Darwinian principle of survival and reproduction of the fitness. Mutation is a second operation in GA. The crossover operation involves the exchange (swap) between two selected bytes (i.e. string of bits) the crossover points are randomly chosen [1].

\section{Structure of the Proposed Genetic Algorithm:}

In this proposed method, the operations of GA (Crossover and mutation) are exploited to produce a new encryption method. This new method was applied to the candidate type of data in this work ( i.e. Images). Many tests are performed to ensure the success of the new proposed encryption method. Some of them are listed in this paper. Then new encryption method is developed to satisfy the following goals, where the variable I be an image, $\mathrm{E}$ is the proposed encryption method and $\mathrm{D}$ is the proposed decryption method.

- Lossless: The encryption process has to be reversible, with perfect reconstruction of the image, $\mathrm{D}(\mathrm{E}(\mathrm{I}))=\mathrm{I}$.

- Secure: The cryptosystem has to be resistant to known attack such that corrections attack. Attacks specific to high redundant messages like images are to be taken into account.

- Complexity: the algorithm has to be based on low-cost operations.

The proposed encryption method consists of the following steps:

1. Initialize algorithm variable, Image I.

2. Initialize values of pseudo random $X_{0}$ and $P$.

3. Generate pseudo random using spatial case depending :

$X_{n}=\left(\left(X_{n-1}\right)^{\wedge 2}+5\right) \bmod p$

4. $E\left(I_{n}\right)=I_{n} \oplus X_{n}, J_{n}=E\left(I_{n}\right)$

5. Using GA crossover for each pixel of Encryption Image $\mathbf{J}$ as shown bellow figure (1).

The proposed decryption method consists of the following steps:

1. Initial value of pseudo -random generator $X_{0}$ and $P$. 
2. Generate pseudo -random using algorithm

$$
X_{n}=\left(\left(X_{n-1}\right)^{\wedge}+5\right) \bmod p
$$

3. Using GA crossover for each pixel of Encryption Image $\mathbf{J}$ as shown bellow figure(2).
4. Decryption using algorithm

$$
D\left(J_{n}\right)=J_{n} \oplus X_{n}
$$

$\mathrm{I}_{\mathrm{n}}=\mathrm{E}\left(\mathrm{J}_{\mathrm{n}}\right)$

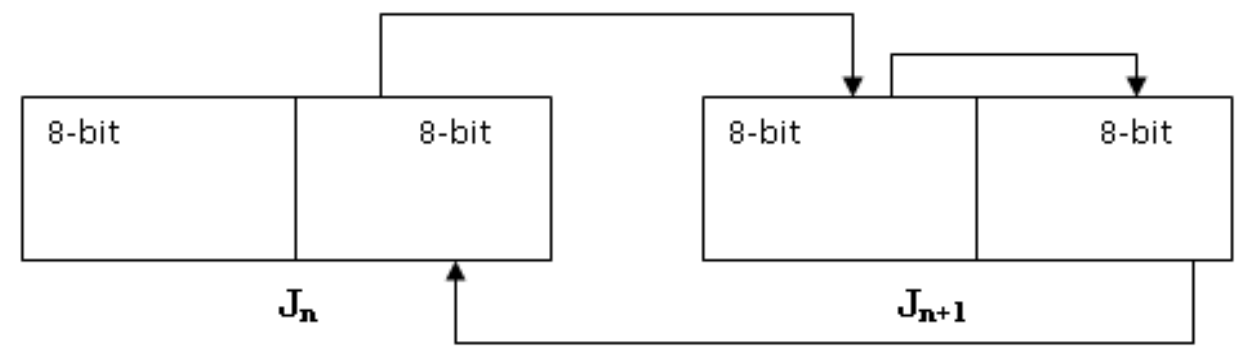

Figure (1):G.A. diagram for encryption.

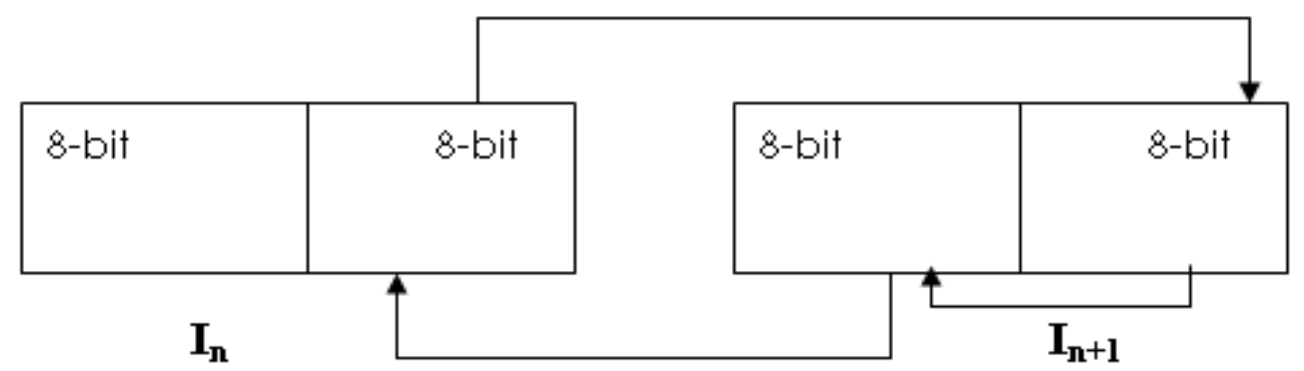

Figure (2):G.A. diagram for encryption.

5. statistical test

In this work, the proposed method pass

four out of the five basic statistical tests to prove the randomness, these tests were:

1. Frequency test (Passed).

2. Serial test (Passed).

3. Poker test (Not Passed).

4. Runs test (Passed).

5. Autocorrelation test (Passed).

\section{$\underline{\text { 5. Experiments }}$}

To perform the proposed encryption method, MATLAB (2007a) was used to write it and applied on different features images, poor and rich details(information). comparing between the image encrypted using the pseudo random generator only, weakness area appear in the encrypted image as shown 




Figure (3): represent the weakness area when depending on the pseudo random generator only. 

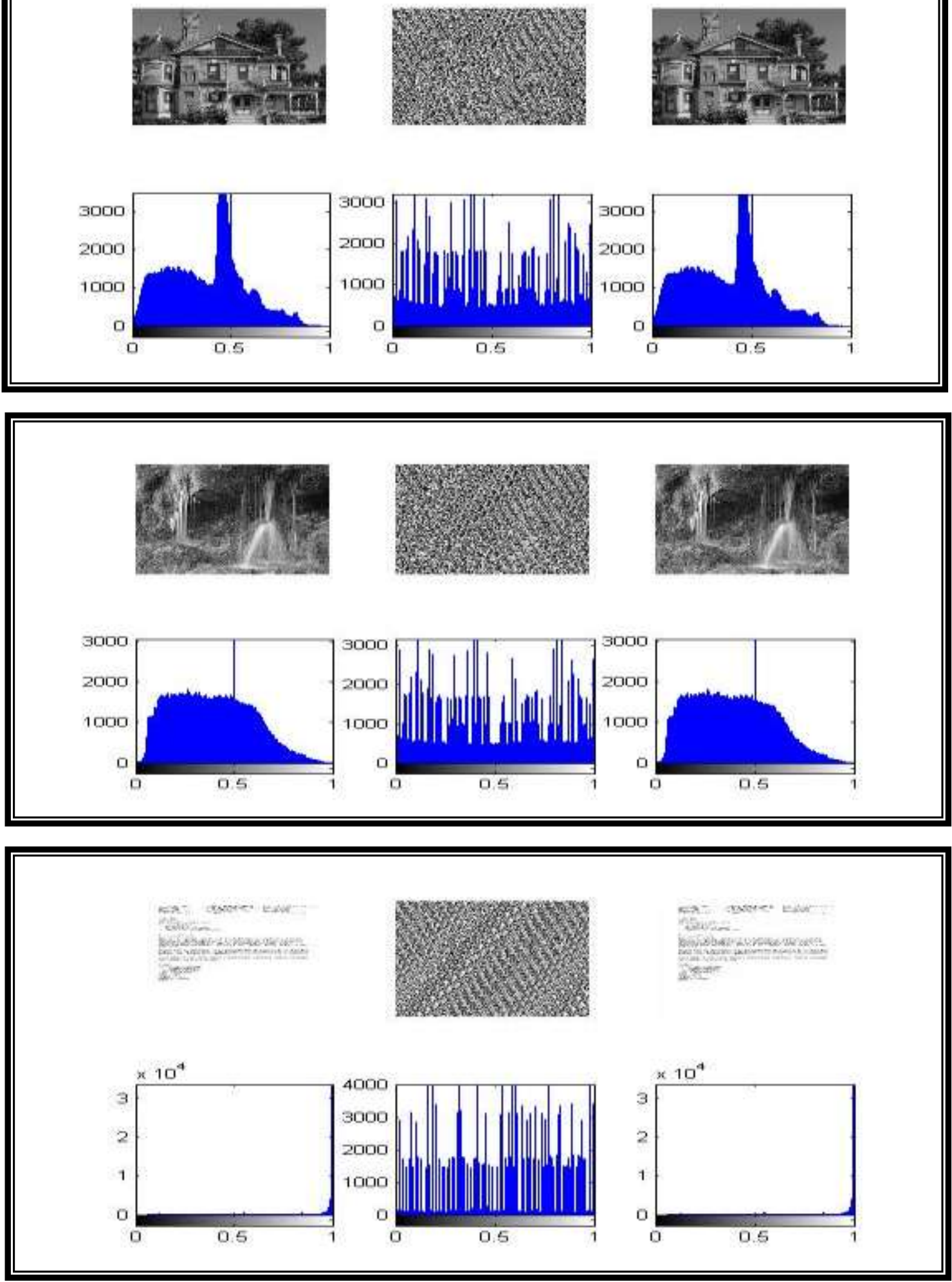

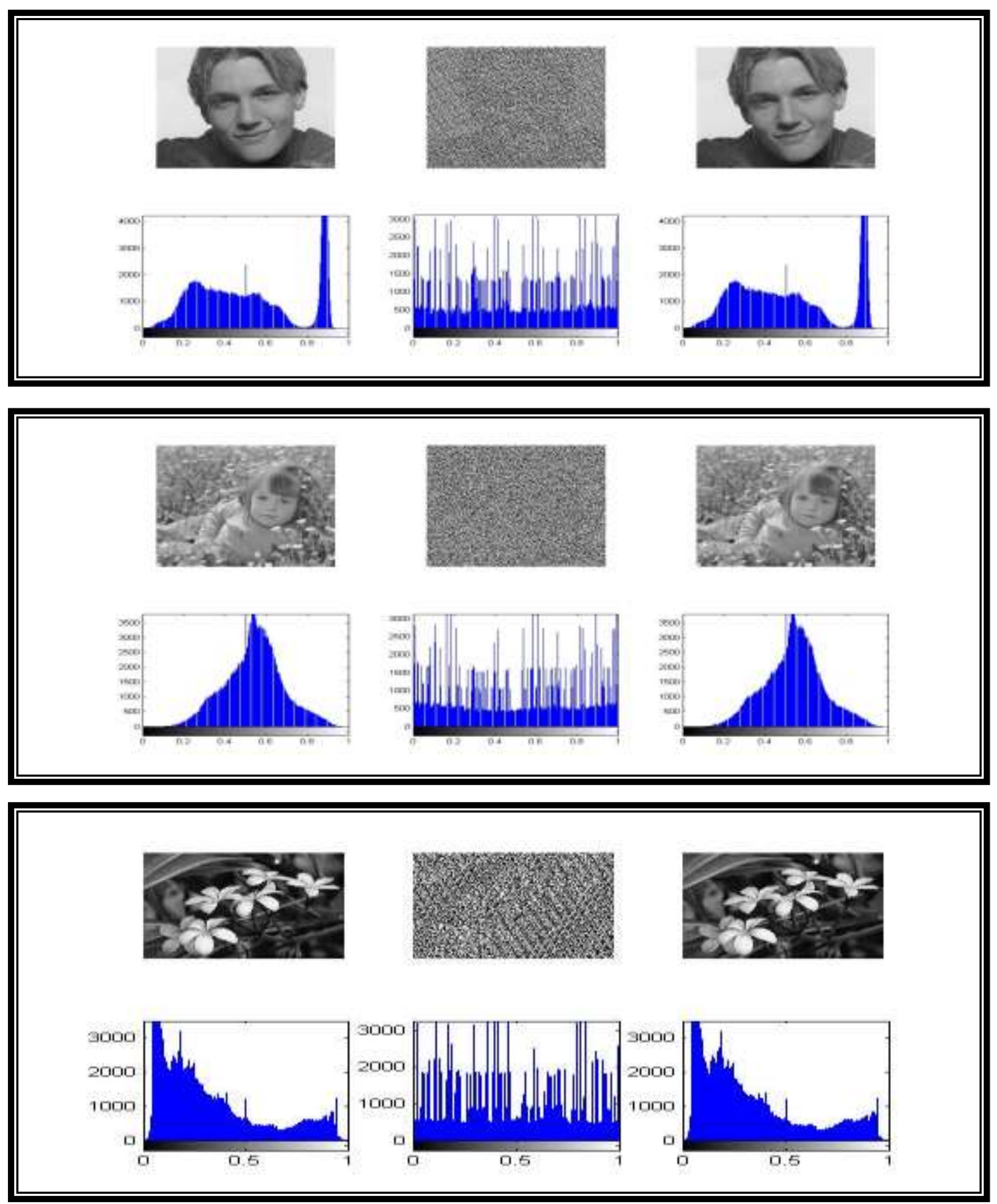

Figure(4):shows first row left column original image, center column encrypted image, right column decrypted image, second row left column original image histogram, center column encrypted image histogram, right column decrypted image histogram. 


\section{Conclusions}

In this paper, we have presented a new scheme for image encryption method, the proposed method depending on merging Pseudo Random Generator with Crossover Genetic Algorithm, the new method is a lossless method and it has a high degree of the complexity with short time processing (few minutes). Additionally, using cryptography only the encrypted image has contain weakness area(contains information lead to break the image encryption) as shown in figure(3), but with G.A. produce high encrypted image as shown in figure(4).

\section{References}

1. M.A.F. Al-Husainy,2006, " image encryption using genetic algorithm", Information Technology Journal 5(3):516519.

2. J.Daemen and V.Rijmen, 2002, "The Design of Rijndael, Advanced
Encryption Standard", Springer-Verlag,Berlin.

3. R.L. Rivest, A. Shamir, and L. Adleman,1978, " A method for obtaining digital signatures and public key cryptosystems", Comm. ACM Vol. 21(2),pp.120-126.

4. R. Stinson, Douglas,1995," Theory and Practice", CRC Press.

5. M.Wenbo,2004,"Modren

Cryptography: Theory and Practice", Publisher Hall PTR, Copyright: Hewlett Packard.

6. D.E. Goldberg, 1989, " Genetic algorithms in search optimization \& Machine learning", Addison- Wesley.

7. Ibrahiem M.M. El-Emary and Mona M. Abd El-Kareem,2008, ,On the Application of Genetic Algorithms in Finger Prints Registration" World Applied Sciences Journal 5(3):276-281. 